\title{
Bericht über das 38. Kriminologische Kolloquium der südwestdeutschen und schweizerischen Kriminologischen Institute
}

\author{
Horst Beisel, Dieter Dölling und Dieter Hermann
}

\begin{abstract}
Vom 5. bis 7. Juli 2002 fand im Bildungszentrum Schloss Flehingen des Landeswohlfahrtsverbandes Baden das 38. Kolloquium der südwestdeutschen und schweizerischen Kriminologischen Institute statt. Veranstalter war das Institut für Kriminologie der Universität Heidelberg. Es wurden acht Forschungsprojekte des Heidelberger Instituts vorgestellt.
\end{abstract}

Christian Laue referierte über den Beitrag der Biowissenschaften zu Kriminalitätserklärungen.

Dabei wurde zunächst der im Vergleich zu den Sozialwissenschaften grundlegend unterschiedliche Forschungsansatz der Human- und Biowissenschaften betont. Während es in der Sozialwissenschaft um die Erklärung gesellschaftlicher Phänomene geht, stehen in der Medizin und Biologie die klinischen, auf Therapie ausgerichteten Anforderungen im Mittelpunkt. Das führt in diesen Wissenschaften zu einer anderen Forschungsmethodik, die den Ansprüchen der empirischen Sozialforschung nur selten genügt. Darüber hinaus besteht der Forschungsgegenstand nicht in Kriminalität, sondern allgemeiner in abweichenden Verhaltensweisen, die beim Patienten oder seiner Umwelt Leiden erzeugen. Die Aufgabe einer biowissenschaftlich interessierten Kriminologie besteht daher primär darin, die Verallgemeinerungsfähigkeit der zahlreichen, vor allem in klinischen Untersuchungen gewonnenen Einzelergebnisse zu überprüfen.

Es wurden im Weiteren einige Beispiele der (vor allem im anglo-amerikanischen und skandinavischen Raum) sehr regen biowissenschaftlichen Forschung aus den Bereichen Neuroendokrinologie, Neurotransmitter und Psychophysiologie dargestellt. In der Neuroendokrinologie, also der Lehre von den Hormonen, stand insbesondere Testosteron im Verdacht, verantwortlich für Kriminalität, vor allem Gewaltkriminalität zu sein. Ein solch allgemeiner Zusammenhang lässt sich allerdings empirisch nicht belegen. Bei den Neuro- 
transmittern, also Botenstoffe für die Weiterleitung von Reizen im Nervensystem, wird insbesondere dem Serotonin eine kriminogene Wirkung zugemessen. Tatsächlich belegen viele Studien eine Korrelation zwischen Serotonin und Aggression bzw. Persönlichkeitsstörungen. Ursache-Wirkungs-Zusammenhänge erscheinen allerdings noch weitgehend ungeklärt. Die Psychophysiologie beschäftigt sich mit dem vegetativen Nervensystem und der menschlichen Fähigkeit der Reizverarbeitung. Die Ausgangsthese lautet, dass Personen mit einer verminderten Basisreaktivität über eine schwächere Fähigkeit zu konditioniertem Vermeidelernen verfügen und gleichzeitig ein erhöhtes Reizbedürfnis haben (sensation seeking). Diese körperliche Besonderheit führe, so die weitere These, zu abweichendem Verhalten und Kriminalität. Auch in diesem Bereich erbringt die empirische Überprüfung bisher eher enttäuschende Ergebnisse. Das mag aber daran liegen, dass die Ausgangsthese noch zu einfach und nur eindimensional ist.

Zuletzt behandelte Laue die von der „herrschenden“ Kriminologie vorgebrachten Haupteinwände gegen eine biowissenschaftlich orientierte Kriminologie: Dazu gehören die methodischen Schwächen bei der empirischen Forschung, die oftmals doktrinären Schlussfolgerungen und die Gefahr der Stigmatisierung bzw. eines latenten oder unverhohlenen Rassismus. Für alle Einwände lassen sich Beispiele finden. Allerdings ist die neuere biowissenschaftlich orientierte Kriminologie in den letzten Jahre zunehmend sensibel geworden für ihre Schwächen und Gefahren. Bei sensibler Handhabung können Biowissenschaften durchaus zur Erklärung bestimmter Kriminalitätsphänomene beitragen. Die Kriminologie sollte sich dem nicht grundsätzlich verschließen. 
Horst Beisel stellte in dem Beitrag Reaktion und Kooperation von Polizei, Staatsanwaltschaft und Jugendamt bei Kinderdelinquenz Ergebnisse einer Hellfeldstudie vor und ging der Frage der institutionellen Bearbeitung eines Tatverdachts gegen Kinder nach. Die Studie basiert auf allen im Jahr 1998 polizeilich erfassten und bei der Staatsanwaltschaft Mannheim eingegangenen Tatverdachtsfällen gegen strafunmündige Kinder im Stadtgebiet Mannheim.

Relevante Hintergrundinformationen zur Kinderkriminalität in Mannheim sind, dass 1,4 \% aller in Mannheim lebenden Kinder im Untersuchungsjahr mit einem Tatverdacht erfasst wurden, dass die kindliche Auffälligkeit zu 97,1 \% im Wohnstadtteil stattfindet und dass der Ladendiebstahl (67,5\%) das die Kinderdelinquenz beherrschende Delikt darstellt. Die Schäden lassen sich im materiellen wie im immateriellen Bereich dem unteren Schwerespektrum zuordnen.

Der Schwerpunkt der Studie liegt in der Analyse der institutionellen Bearbeitung von Kinderdelinquenz. Der Umgang der Polizei mit tatverdächtigen Kindern ist durch einen relativ häufigen unmittelbarem Kontakt charakterisiert. Die Staatsanwaltschaft stellt diese Fälle zwar grundsätzlich ein, in Einzelfällen werden aber Tatverdachtsinformationen an Vormundschaft- bzw. Familiengerichte und Jugendämter weiter geleitet. Die Merkmale mehrfache Tatverdachtsregistrierung, Begehung schwerer Deliktsformen, vergleichsweise starke körperliche Schädigung von Personen, aber auch soziale Merkmale wie beispielsweise der Sonderschulbesuch eines tatverdächtigen Kindes führen tendenziell zu einer verstärkten Weiterleitungen der Tatverdachtsinformationen seitens der Staatsanwaltschaft. Zudem zeigten sich bei den untersuchten Fällen deutliche Unterschiede in der Mitteilungspraxis der einzelnen Staatsanwälte. 
Die Analyse der jugendamtlichen Praxis war auf die Kenntnis der Tatverdachtsbelastung sowie Art und Umfang der Hilfeformen gegenüber strafunmündigen Kindern und deren Betreuungspersonen konzentriert. Das Jugendamt hatte in der Hälfte aller Tatverdachtsfälle gegen Kinder zumindest grobe Kenntnis von den Vorgängen, während die Staatsanwaltschaft nur in weniger als einem Drittel der Fälle Information an das Jugendamt weiterleitete. Dies belegt, dass das Jugendamt Tatverdachtsinformationen nicht nur über die Staatsanwaltschaft, sondern auch über andere Wege (andere Abteilungen der Jugendhilfe, Kinder, Eltern etc.) erhält. Für den Umgang des Jugendamts mit staatsanwaltschaftlichen Hinweisen auf Kinderdelinquenz konnten zwei Strategien festgestellt werden: Liegt nur ein staatsanwaltschaftlicher Delinquenzhinweis vor, reagieren die Jugendamtsmitarbeiter überwiegend mit einem schriftlichen Beratungsangebot an die Erziehungsberechtigten. Liegen jedoch ergänzende Hinweise oder Erkenntnisse zu dem Kind oder der Familie vor, wird mit den Betroffenen häufiger ein persönlicher Kontakt hergestellt. In den meisten Fällen mit persönlichem Kontakt kommt es $\mathrm{zu}$ formloser Beratungs- oder Hilfegewährung nach dem KJHG.

Die Kooperation zwischen Polizei, Staatsanwaltschaft und Jugendamt ist durch eine erhebliche zeitliche Verzögerung bei der Weiterleitung von Tatverdachtsinformationen von der Staatsanwaltschaft zum Jugendamt gekennzeichnet. Die Auswertung der Weiterleitungszeiträume ergab einen etwa dreimonatigen Zeitraum zwischen Tatverdacht und Bekanntwerden des Vorfalls beim Jugendamt. In einigen Fällen mit massiven delinquenten Auffälligkeiten von Kindern scheint ein Verbesserungspotential in der Mitteilungspraxis vorhanden zu sein. Aus der Untersuchung konnten Vorschläge für die Praxis abgeleitet werden. 
Jörg Dittmann behandelte in seinem Beitrag über Input und Output der Strafjustiz die Frage nach dem Einfluss der Fallbelastung auf die Erledigungspraxis an Landgerichten. Das Vortragsthema bildet einen Teil seiner Dissertation, in der eine systemtheoretische und organisationssoziologische Theorie der Erledigungspraxis von Staatsanwaltschaften und Gerichten entwickelt werden soll.

Im ersten Teil des Referats wurden Hypothesen über die Beziehung zwischen In- und Output der Strafjustiz vorgestellt. Der Output von Landgerichten wurde als Anzahl der innerhalb eines Geschäftsjahres erledigten Fälle gemessen. Basierend auf organisationssoziologischen und systemtheoretischen Überlegungen, insbesondere auf Ideen des Ressourcenabhängigkeitsansatzes, wurde angenommen, dass Unterschiede in der Anzahl der erledigten Verfahren zwischen den Landgerichten sowohl durch das unterschiedliche Fallaufkommen als auch durch unterschiedliche interne Ressourcenausstattung und Differenzen in der Fallstruktur (hoher vs. niedriger Anteil an ressourcen- und zeitaufwändigen Wirtschaftsstrafsachen) zustande kommen. Für das Funktionieren des Justizsystems ist es notwendig, dass Gerichte flexibel sind und sich unterschiedlichem Input anpassen, denn die Nicht- bzw. verspätete Erledigung von Strafverfahren würde die Wiederherstellung des Rechtsfriedens gefährden. Somit kann postuliert werden, dass an Gerichten mit hoher Fallbelastung die Erledigungsart stärker „ökonomisch“ ausgerichtet ist: Verfahren werden durch die Staatsanwaltschaft häufiger eingestellt und die Gerichte erledigen - trotz gleichbleibender Ressourcenausstattung mehr Verfahren als Gerichte mit niedriger Fallbelastung. Demnach müsste die Einstellungspraxis der Staatsanwaltschaft sowie die Erledigungsfrequenz der Gerichte jeweils vom Fallaufkommen abhängen. Außerdem wird untersucht, inwieweit eine erhöhte Selektionspraxis der Staatsanwaltschaft die gerichtliche Fallstruktur verändert.

Die Überprüfung dieser Hypothesen erfolgte mit Falldaten aus Aktenanalysen zu erstinstanzlichen Verfahren an vier für Deutschland repräsentativen Landgerichten, mit Strukturdaten zu den Landgerichten und Landgerichtsbezirken der Bundesrepublik Deutschland (jeweils aus dem Jahre 1994) und mit Längsschnittdaten zur Geschäftsentwicklung an Landgerichten in Bayern für die Jahre 1990 bis 1997. Die Daten wurden vornehmlich im Rahmen des im 
Auftrag des Bundesministeriums der Justiz vom Heidelberger Institut für Kriminologie durchgeführten Projekts zur Dauer von Strafverfahren vor den Landgerichten (Dölling et al. 2000) erhoben.

Die Informationen über Landgerichte und Landgerichtsbezirke wurden bei den Landeskriminalämtern, Statistischen Landesämtern und den Präsidenten der Landgerichte eingeholt. Um die unterschiedlich großen Landgerichte miteinander vergleichen zu können, wurde das Erledigungsvolumen zu dem Personalvolumen des jeweiligen Landgerichts in Beziehung gesetzt. Dafür wurde die Zahl der erledigten Verfahren an den Landgerichten mit der Anzahl der Richter gewichtet, die an diesem Landgericht in Strafsachen eingesetzt wurden.

Im Erledigungsvolumen pro Richter eines Landgerichts bestehen im Untersuchungsjahr 1994 große Unterschiede zwischen den Landgerichten, sowohl was die Verfahren der ersten als auch der zweiten Instanz angeht. Im Vergleich zur durchschnittlichen Zahl der erledigten Verfahren der ersten Instanz, die im Mittel 10,1 Verfahren pro Richter betrug, lag die Erledigungsfrequenz für zweitinstanzliche Fälle mit 32,7 Berufungsverfahren pro Richter wesentlich höher. Bemerkenswert ist auch, dass - selbst unter Kontrolle der Fallstruktur - an großen Gerichtsbezirken die Zahl der pro Richter erledigten Verfahren geringer ist als an Gerichten mittlerer und kleinerer Größe. Dies gilt sowohl für Verfahren der ersten als auch der zweiten Instanz.

Für die Überprüfung der Hypothesen zur Entlastungsfunktion der Staatsanwaltschaft sowie der veränderten Fallkonstellationen aufgrund erhöhter Selektionstätigkeit der Staatsanwaltschaft wurde ebenfalls auf die Daten über Landgerichte und Landgerichtsbezirke zurückgegriffen. Die Datenanalyse kommt zu folgenden Befunden: (1) Ist die Kriminalitätsbelastung, gemessen an der Zahl der polizeilich registrierten Fälle pro 100.000 Einwohner, eines Landgerichtsbezirks verhältnismäßig hoch, stellt die Staatsanwaltschaft dort mehr Verfahren ein als in Landgerichtsbezirken mit niedriger Kriminalitäts- 
belastung und vermeidet somit einen hohen Geschäftsanfall bei Gericht. Zwischen der lokalen Kriminalitätsbelastung und der gerichtlichen Fallbelastung kann deshalb auch kein Zusammenhang festgestellt werden. (2) Die Erledigungsfrequenz an den Gerichten mit großer Fallbelastung ist überdurchschnittlich hoch. Gerichte mit einer pro Richter hohen Zahl an anhängigen Verfahren zu Beginn sowie an Neuzugängen während des Geschäftsjahres erledigen demzufolge mehr Verfahren als weniger fallbelastete Gerichte. (3) Eine ausgeprägte Selektionstätigkeit der Staatsanwaltschaft, gemessen am hohen Anteil der Einstellungen ohne Auflage an allen Erledigungsarten (Einstellung, Strafbefehlsantrag, Anklage usw.), führt zu einer Konzentration schwerer Fälle an den jeweiligen Gerichten.

Anhand von Längsschnittdaten über die Geschäftsentwicklung am Landgericht München I wird deutlich, dass die Erledigungsfrequenz bei Gericht häufig mit der Fallentwicklung einhergeht. Mit einem Inputanstieg nimmt auch die Zahl der erledigten Verfahren zu. Umgekehrt sinkt die Zahl der erledigten Verfahren mit einem Rückgang der gerichtlichen Fallzahl. Allerdings können Inputveränderungen durch Variation der Erledigungsfrequenz des Gerichts nicht vollständig kompensiert werden.

Insgesamt liefert die Analyse von Querschnitts- und Längsschnittdaten zu den Landgerichten und Landgerichtsbezirken Einblicke in Regelmäßigkeiten der Arbeitsbewältigung der Strafjustiz. 
Reinhart Ensslin referierte über sein Dissertationsvorhaben zu normativdogmatischen Problemen der spezialpräventiven Strafzumessung nach dem $S t G B$. Die Fragestellung ist auf die Entwicklung eines dogmatisch korrekten und praktikablen Entscheidungsverlaufs für die Anwendung von $\S 46$ I 2 StGB gerichtet.

Nach der herrschenden Schuldrahmentheorie läuft die Strafzumessung in drei Schritten ab: Nach der Ermittlung des gesetzlichen Strafrahmens folgt die Festlegung des Schuldrahmens innerhalb des Strafrahmens nach $§ 46$ I 1 StGB. Im dritten Schritt erfolgt die Festlegung von Strafart und Strafhöhe. Auf dieser Ebene müssen präventive Überlegungen berücksichtigt werden, und hier ist damit auch die Anwendung des § 46 I 2 StGB angesiedelt.

Aufgrund der Verknüpfung der Begriffe „Täter“, „Wirkungen“, und „Gesellschaft" ist bereits aus dem Wortlaut der Norm ersichtlich, dass Resozialisierung und die Vermeidung der Entsozialisierung wesentliche Ziele der Norm sind. Entsozialisierung ist die Gefahr der Herauslösung des Täters aus einer erlangten sozialen Stellung. Will man Resozialisierung erreichen, dann muss man Entsozialisierung vermeiden. Da § 46 I 2 StGB eine zukunftsorientierte Aussage trifft, erfordert die Vorschrift eine Prognose. Bestandteile einer solchen sind nach Frisch (1983, S. 12) die Prognosebasis oder Tatsachengrundlage und ein allgemeiner theoretischer Satz oder eine „Gesetzmäßigkeit”, die die Grundlage mit dem Prognoseergebnis verknüpft. Die Gesetzmäßigkeit ist das Produkt von erfahrungswissenschaftlich erlangtem Wissen. Entsprechend diesen Bestandteilen ist auch das Entscheidungsprogramm für $\S 46$ I 2 StGB zu sehen.

Als Erstes wird eine Prognosebasis oder Tatsachengrundlage benötigt. Dies können zunächst die Strafzumessungstatsachen sein. Grundlage ist hier die Bestimmung des $\S 46$ II 2 StGB, die Strafzumessungstatsachen exemplarisch nennt. Berücksichtigungsfähig sind z.B. Faktoren wie die berufliche Stellung, deren Verlust vermieden werden soll, oder die sozialen Bindun- 
gen an eine Familie, die eine Rückkehr in ein „normales“ Leben nach der Strafe erleichtern können. Ein Sonderfall ist die Berücksichtigung der Aussetzbarkeit der Strafe. Obwohl die Schuldrahmentheorie eine Trennung der Strafzumessung im engeren Sinne von den Folgeentscheidungen vorschreibt, findet sich in Rechtsprechung und Literatur Zustimmung dazu, dass die Aussetzungsfähigkeit der zu verhängenden Strafe bereits auf der Ebene der Strafhöhenentscheidung, also im Rahmen der spezialpräventiven Entscheidung nach § 46 I 2 StGB, innerhalb des Schuldrahmens berücksichtigt werden darf. Dem stehen keine Bedenken entgegen, weil Strafaussetzung zur Bewährung ebenso wie § 46 I 2 StGB durch die Resozialisierung motiviert ist.

Für die Prognose benötigt man Kenntnisse, die unter Zugrundelegung von allgemeinen, wissenschaftlich fundierten und empirisch abgesicherten Gesetzmäßigkeiten die Strafzumessungstatsachen mit einer Folge - der späteren Sanktionsentscheidung - zumindest mit einem hohen Maß an Wahrscheinlichkeit verknüpfen. Die Strafzumessungstatsachen tragen dabei mit der Bewertungsrichtung einen Teil der Gesetzmäßigkeiten bereits in sich: Für die Strafart ist z.B. festzustellen, dass Tatsachen, die ein hohes Maß an sozialer Integration kennzeichnen, eher für eine ambulante Sanktion und damit für die Geldstrafe sprechen. Genaueres Wissen zu liefern ist Aufgabe der Erfahrungswissenschaften. In der Dissertation wurden Untersuchungen ausgewertet, die die Rückfälligkeit von Erwachsenen im Zusammenhang mit Freiheitsstrafe, Geldstrafe und Modifikationen wie der Sozialtherapie zum Inhalt hatten. Dabei hat sich ergeben, dass es zwar Wissen über grobe Zusammenhänge sowohl zwischen Strafzumessungstatsachen und Legalbewährung als auch zwischen Strafarten und Legalbewährung gibt. Jedoch können aus diesen groben Ergebnissen keine verlässlichen Aussagen abgeleitet werden, die eine Entscheidung sicher bedingen. Solche Unsicherheiten der Prognose werden von Streng (1995, S. 109) anhand des sogenannten Problems des Mittelfeldes dogmatisch beschrieben. Eine Lösung lässt sich für § 46 I 2 StGB aus der Norm selbst ableiten. Da sie die Vermeidung der Entsozialisierung vorschreibt 
und eine Strafe jenes Risiko immer mit sich bringt, kann aus $§ 46$ I 2 StGB die Anweisung abgelesen werden, in unklaren Fällen die Strafe bis an den unteren Rand des Schuldrahmens zu mildern. Dabei besteht allerdings das Risiko, dass das ganze System der Schuldrahmentheorie, die ja gerade Raum für eine Variation der Strafen bieten will, ad absurdum geführt werden könnte und es damit zu einer Aushöhlung der gesamten Strafzumessungsnorm des $§ 46$ StGB kommen kann.

Die Vorschläge zur Verbesserungen der Prognose im Rahmen des § 46 I 2 StGB sind: Auf der Ebene der Strafzumessungstatsachen könnte der Ausbau der Gerichtshilfe zu einer Wissenserweiterung beitragen. Zudem würde die Einführung einer in Abschnitte zur Tat- und zur Rechtsfolgefragen geteilten Hauptverhandlung zu einer stärkeren Berücksichtigung von Überlegungen zur Strafzumessung führen. Schließlich könnte die Methode der idealtypischvergleichenden Einzelfallanalyse nach Göppinger (1985) und Bock (1994) die Erfassung und Einstufung der Täterperson aus einem kriminologisch fundierteren Blickwinkel ermöglichen.

Dieter Hermann stellte Konzeption und erste Ergebnisse einer Metaanalyse empirischer Abschreckungsstudien vor. Der Vortrag war auf die Frage konzentriert, weshalb die Resultate solcher Studien widersprüchlich sind. Das Projekt wird von Dieter Dölling, Horst Entorf und dem Referenten durchgeführt.

Die negative Generalprävention ist zwar eine normative Theorie zur Rechtfertigung staatlichen Strafens. Der Ansatz muss jedoch, wie alle funktionalen Straftheorien, empirisch belegt werden, denn Abschreckungsmaßnahmen verlieren ihre Legitimation, wenn die Wirkungslosigkeit oder gar Kontraproduktivität solcher Maßnahmen nachgewiesen werden kann. Die empirischen Untersuchungen zu der Frage nach der abschreckenden Wirkung von Strafen sind 
jedoch widersprüchlich. Während beispielsweise Lamnek (1994, S. 235) die Ergebnisse einschlägiger Studien so zusammenfasst, dass „Strafandrohung, Strafschwere und objektive Sanktionswahrscheinlichkeiten praktisch keinen Einfluss auf das Begehen von Straftaten haben“, kommt Albrecht (1980, S. 320) zu dem Schluss, dass Abschreckungseffekte zumindest bei Personen mit schwacher Normbindung nachweisbar sind. Bishop (1984, S. 311) folgert aus den Ergebnissen ihrer Studie sogar eine generelle Gültigkeit des Abschreckungsmodells. Diese unterschiedlichen Ansichten spiegeln die Forschungslage wider. Eisele (1999) beispielsweise hat in einer qualitativen Metaanalyse 28 elaborierte Studien zur negativen Generalprävention berücksichtigt. Davon bestätigen 9 Studien die Abschreckungshypothese, 9 Studien widerlegen sie. In 10 Studien wird die ursprüngliche Hypothese modifiziert und beispielsweise die Abhängigkeit der Abschreckungswirkung von Rahmenbedingungen postuliert.

Durch eine Metaanalyse sollen Ursachen für die Unterschiede in den Untersuchungsergebnissen gefunden werden. Dazu ist es notwendig, die einschlägigen Untersuchungen durch eine Erfassung von Untersuchungsanordnung, Methode der statistischen Analyse und Untersuchungsergebnissen zu beschreiben. Anschließend kann in quantitativen statistischen Analysen nach Beziehungen zwischen diesen Merkmalen gesucht werden. Zudem sollen Einzelergebnisse mehrerer Untersuchungen $\mathrm{zu}$ einer Fragestellung durch metaanalytische Verfahren zusammen gefasst werden, so dass im Vergleich zu Einzelstudien eine validere Schätzung von Abschreckungseffekten möglich ist.

In einer Voruntersuchung wurden 24 Studien berücksichtigt. Das Ziel dieser Voruntersuchung war es, Probleme der geplanten Metaanalyse bereits im Vorfeld aufzudecken sowie erste Analysen über die Beziehungen zwischen Methoden und Ergebnissen von Untersuchungen durchzuführen. 
Ein Problem der Metaanalyse ist, dass die empirischen Studien zur Abschreckungsforschung von unterschiedlicher Qualität sind. Es erscheint sinnvoll, Studien mit Artefakten auszuschließen. Artefakte sind vermutlich nicht einfach zu erkennen, es sei denn, in anderen Untersuchungen gibt es Hinweise. Dies soll durch die Einbeziehung des Diskussionskontextes von Untersuchungen erfasst werden. Bei Untersuchungen mit ungewöhnlichem Design könnte zudem eine Experteneinschätzung berücksichtigt werden.

Die Analysen von 24 empirischen Abschreckungsstudien führten zu folgenden Ergebnissen: (1) Unterschiedliche Operationalisierungen von unabhängigen und abhängigen Variablen sind eine Ursache für Diskrepanzen in den Ergebnissen. (2) Durch die Zusammenfassung von Items bei abhängigen und unabhängigen Variablen können Abschreckungseffekte deutlicher aufgezeigt werden als durch Analysen mit Einzelvariablen. (3) Die Korrelationen zwischen perzipiertem Abschreckungsniveau und selbstberichteter Delinquenz sind in Befragungen mit mehreren Erhebungswellen etwas niedriger als in Querschnittsstudien. (4) In Untersuchungen mit aggregierten Daten werden Abschreckungseffekte häufiger nachgewiesen als in Untersuchungen mit Individualdaten. Die üblichen Schlussfolgerungen aus den letzten beiden Punkten sind, dass Längsschnittstudien elaborierter und zuverlässiger seien als Querschnittstudien, weil sie die Beziehung zwischen Ursache und Wirkung korrekt abbilden würden, und dass Analysen mit Befragungsdaten elaborierter seien als Untersuchungen mit Aggregatdaten, weil bei Befragungen auch die nicht registrierte Kriminalität berücksichtigt werde. Allerdings ist auch eine alternative Interpretation der Ergebnisse möglich:

Die Folgerung, bei einer Analyse mit individuellen Querschnittsdaten würden generalpräventive Effekte überschätzt und mit einer Panelstudie könnten zuverlässigere Ergebnisse erzielt werden, ist nicht zwingend. Genau genommen sind beide Untersuchungsformen nicht in der Lage, Entscheidungs- 
prozesse korrekt abzubilden. Geht man davon aus, dass das perzipierte Abschreckungsniveau genau in dem Augenblick handlungsrelevant ist, in dem die Entscheidung über die Handlung getroffen wird, kann durch keine der beiden Untersuchungsarten dieses Merkmal erfasst werden. In einer Querschnittsuntersuchung wird das Abschreckungsniveau nach und in einer Längsschnittsuntersuchung vor dem relevanten Zeitpunkt gemessen. Sowohl durch Quer- als auch durch Längsschnittsstudien ist demnach eine präzise Messung des Abschreckungsniveaus zum Entscheidungszeitpunkt nicht möglich. Durch formal-statistische Überlegungen kann gezeigt werden, dass sowohl Längsals auch Querschnittsuntersuchungen in der Regel zu verzerrten Schätzungen führen. Analysen mit Paneldaten führen immer zu einer Unterschätzung des Abschreckungseffekts, während Analysen mit Querschnittsdaten sowohl zu einer Über- als auch Unterschätzung führen können.

Das Ergebnis, dass in Untersuchungen mit aggregierten Daten häufiger Abschreckungseffekte nachgewiesen werden als in Studien mit Individualdaten kann mit unterschiedlichen Fehlerniveaus zusammenhängen. Aggregatdaten sind mit geringeren zufälligen Messfehlern behaftet als subjektive Individualdaten. Solche Messfehler haben einen erheblichen Einfluss auf die Schätzung statistischer Parameter. Dies kann durch eine Reanalyse der Daten der Untersuchung von Bishop $(1982,1984)$ verdeutlicht werden. Die Anwendung eines Analyseverfahrens, mit dem die Auswirkungen zufälliger Messfehler auf die Schätzung von Korrelationskoeffizienten kompensiert werden können (Strukturgleichungsmodelle), führt zu einer Korrelation von -0,56 zwischen perzipiertem Entdeckungs- und Sanktionsrisiko und selbstberichteter Delinquenz. Im Vergleich dazu ist die unbereinigte Korrelation nur etwa halb so groß. Zufällige Messfehler können sich somit erheblich auf Untersuchungsergebnisse auswirken, wenn diese nicht durch statistische Verfahren kompensiert 
werden. Somit könnten die Ergebnisse von Abschreckungsstudien mit Aggregatdaten zuverlässiger als entsprechende Untersuchungen mit Individualdaten sein.

Insgesamt gesehen sprechen die vorläufigen Ergebnisse der Metaanalyse nicht für eine vollständige Falsifikation des Abschreckungsansatzes, sondern eher für eine Modifikation. Möglicherweise ist die Wirkung von Abschreckungsmaßnahmen bei bestimmten Personengruppen oder Handlungen eingeschränkt. Außerdem scheinen nicht alle bisher entwickelten Untersuchungskonzepte in gleichem Maß in der Lage zu sein, Abschreckungseffekte nachzuweisen. Hier soll die geplante Metaanalyse Unterschiede aufzeigen und dadurch zur Entwicklung eines geeigneten Untersuchungsdesigns beitragen.

Anja Lindrath berichtete über das Projekt Chance, einer neue Form des Strafvollzugs an jungen Gefangenen in Baden-Württemberg. Ausgangspunkt des Dissertationsprojekts ist die Auswertung von Angeboten, mit denen sich private Dienstleister aus dem Bereich der Jugendhilfe um die Durchführung des Modellprojektes beworben haben. Es ist das Ziel der Arbeit, mit Hilfe dieser Angebote und einer Literaturauswertung Standards für den Jugendstrafvollzug an jugendlichen Tätern herauszuarbeiten.

Bei dem Projekt Chance handelt es sich um ein Modellprojekt für den Strafvollzug an Tätern zwischen 14 und 17 Jahren. Rechtsgrundlage ist $\S 91$ Abs. 3 JGG. In dem Projekt sind Organisationsform und Finanzierung des Jugendstrafvollzugs neu gestaltet. Insbesondere wird der Jugendstrafvollzug in freier und privater Form durchgeführt. Das Projekt Chance wird eine Einrichtung der Jugendhilfe sein, die Heimaufsicht erfolgt durch den zuständigen Landeswohlfahrtsverband. Träger der Einrichtung ist der gemeinnützige Verein „Projekt Chance e.V.“, der im Juli 2001 mit Mitgliedern aus Justiz, Jugendhilfe und Wirtschaft gegründet wurde. Vorstandsvorsitzender ist der (ehemalige) Justizminister Ulrich Goll. Ein privater Dienstleister soll im Auftrag des Vereins Projekt Chance e.V. eine Einrichtung betreiben, in 
der mit eigenem Personal ein „Strafvollzug“ mit Erziehungsprogramm angeboten wird. Mit dem Träger können kostendeckende Pflegesätze abgerechnet werden.

Um einen geeigneten Dienstleister für die Modelleinrichtung zu ermitteln, führte der Verein im August 2001 eine Ausschreibung durch. Auf die Ausschreibung bewarben sich 13 Dienstleister mit Erfahrungen aus dem Bereich der Jugendhilfe. Im März 2002 fiel die Entscheidung zugunsten des Christlichen Jugenddorfwerks Deutschland e.V. (CJD). Die Modelleinrichtung soll in der Gemeinde Creglingen im Kloster Frauental untergebracht werden. Gründe für die Schaffung der Modelleinrichtung sind vor allem die Zunahme der Zahl der jugendlichen Strafgefangenen in den letzten Jahren, das Bestreben, die jüngeren Gefangenen von den älteren zu trennen sowie eine Übersicherung zu vermeiden. Den Jugendlichen soll ein altersgemäßes Erziehungsprogramm angeboten werden, Jugendstrafvollzug und Jugendhilfe sollen durch das Projekt stärker vernetzt werden.

Die eingereichten Angebote enthalten vielfältige und differenzierte Ideen zur Gestaltung des Strafvollzugs an jungen Gefangenen. Um diese interessanten Ideen nicht ungenutzt zu lassen, sollen die Angebote aller an der Ausschreibung teilnehmenden Anbieter bezüglich verschiedener Kriterien ausgewertet und analysiert werden.

Die Auswertung der Angebote soll folgende Fragen beantworten: (1) Auf welchen Ansätzen bzw. Konzepten basieren die Erziehungsprogramme der Anbieter? Beispiele hierfür sind etwa das Prinzip der gerechten Gemeinschaft (Just Community) oder der Ansatz der Glen Mills Schools. (2) Welche Ziele werden mit dem Erziehungsprogramm angestrebt? Hierunter fallen beispielsweise die Rückfallverhinderung als zentrales und durch $\S 91$ Abs. 1 JGG vorgegebenes Ziel sowie die Förderung von bestimmten Fähigkeiten, Einstellungen und Bindungen. Weiterhin soll gefragt werden, ob Tatverarbeitung oder die Verhinderung von subkulturellen Strukturen ein angestrebtes Ziel darstellt. (3) Welche Fähigkeiten der jugendlichen Teilnehmer sollen gefördert werden? Hierzu zählen etwa die Förderung von Fähigkeiten im Leistungsbereich, also Schule und Beruf, bei der Alltagsbewältigung, bei verbaler 
und nonverbaler Kommunikation und Selbst- und Fremdwahrnehmung, zudem die Steigerung sozialer Kompetenz sowie sportlicher und künstlerischmusischer Fähigkeiten. (4) Welche Einstellungen der Jugendlichen sollen gefördert werden? Hierzu gehören insbesondere Steigerungen von Normakzeptanz, Verantwortungsbereitschaft und Empathie der Jugendlichen. (5) Soll auf Bindungen in den Bereichen Schule, Beruf und Freizeit Einfluss genommen werden und sollen Bindungen zu Bezugspersonen gefördert werden? (6) Welche Methoden werden vorgeschlagen, um die angestrebten Ziele zu erreichen? Dazu gehören beispielsweise Vorgaben über die Unterbringung der Jugendlichen, die Planung des Tagesablaufs, Angebote zu Schulausbildung, Berufsfindung, Ausbildung und Arbeit, aber auch soziale Trainingskurse, Anti-Aggressionstraining sowie der Einsatz von Bezugspersonen für den Jugendlichen (Ehrenamtliche, Paten). (7) Wie transparent ist die Behandlung für die Jugendlichen? (8) Wie werden Wiedereingliederung und Nachbetreuung, also die Zeit nach der Entlassung vorbereitet? (9) Mit welchen weiteren Kriterien können die Angebote charakterisiert werden? Dazu zählen u.a. der Hintergrund und Schwerpunkt der bisherigen Arbeit des Bewerbers, die personelle Ausstattung, die veranschlagten Kosten, die Öffentlichkeitsarbeit, die bauliche Gestaltung der Einrichtung, Infrastruktur und Entfernung zur JVA Adelsheim.

Die Auswertung der Angebote, die in einigen Fällen durch Interviews mit Anbietern ergänzt wurde, ist mittlerweile abgeschlossen. Außerdem ist eine Umfrage bei den Landesjustizministern der anderen Bundesländer geplant, die Klarheit darüber verschaffen soll, ob schon ähnliche Einrichtungen bestehen oder geplant sind. Nach Durchführung der Auswertungen soll eine Diskussion der Ergebnisse im Kontext von Kriminalitätstheorien und Ergebnissen der Wirkungsforschung erfolgen. Am Ende steht das Ziel, aus den gewonnenen Erkenntnissen Standards für den Strafvollzug an jungen Straftätern zu entwickeln. 
Michael Häuser trug Ergebnisse seiner Dissertation über den Gefahrenbegriff im Strafvollzugsgesetz - eine Analyse anhand von Literatur, Rechtsprechung und Praxis der Vollzugsverwaltung - vor. Ein Beispiel aus der Strafvollzugspraxis kann die Relevanz dieser Thematik verdeutlichen: Ein Gefangener im Bundesland A darf einen bestimmten Gegenstand besitzen, im Bundesland B ist der Besitz des Gegenstandes wegen der Gefährdung des Vollzugsziels oder der Sicherheit oder Ordnung der Anstalt verboten, ohne dass sich der Sicherheitsgrad der Anstalten unterscheidet. Die Fragestellung der Studie ist: Gibt es einen einheitlichen Gefahrenbegriff im Strafvollzugsgesetz und wie wird der Gefahrenbegriff in der Strafvollzugspraxis ausgelegt?

Ausgangspunkt der Analyse ist eine Unterscheidung zwischen konkreter und abstrakter Gefahr: „Konkrete Gefahr“ bedeutet eine Sachlage, die bei ungehindertem Fortgang eine Störung mehr oder minder wahrscheinlich erscheinen lässt. „Abstrakte Gefahr“ ist dagegen eine typischerweise kritische Situation, wobei es auf die Gegebenheiten des Einzelfalls nicht ankommt. Von den 35 Normen des Strafvollzugsgesetzes, die sich ausdrücklich auf eine Gefahr beziehen, weisen nach der Literatur 31 einen konkreten Gefahrenbegriff im Sinne der aufgestellten Definition auf. Nur eine Norm lässt sich unter einen abstrakten Gefahrenbegriff fassen ( $§ 19$ StVollzG, Ausstattung des Haftraumes). Zwei Paragraphen weisen sowohl konkrete wie abstrakte Ansätze in verschiedenen Absätzen der jeweiligen Vorschrift auf (§ 22 StVollzG, Einkauf und $\S 180$ StVollzG, Datenschutz). Folglich gibt es keinen einheitlichen Gefahrenbegriff im StVollzG. Sowohl die Wahrscheinlichkeitsmaßstäbe als auch die Gefahrenintensität (d.h. die Schwere der Gefahr) erfahren keine einheitliche Ausgestaltung durch die Literatur, weder in bezug auf das gesamte Strafvollzugsgesetz noch auf einzelne Normen. 
In der Rechtsprechung wird für 18 der 35 Paragraphen mit einem Bezug zum Gefahrbegriff auf eine konkrete Gefahr abgestellt. Drei Normen (§ 33 I 4 StVollzG, Pakete, § 68 II 2 StVollzG, Zeitungen und $\S 70$ II StVollzG, Gegenstände zur Freizeitbeschäftigung) liegt nach der überwiegenden Rechtsprechung ein abstrakter Ansatz zu Grunde. Bei $§ 22 \mathrm{StVollzG}$ (Einkauf) finden beide Einordnungen Verwendung. Zu § 19 StVollzG (Ausstattung Haftraum) ist die Auslegung uneinheitlich, so dass insgesamt fünf Vorschriften zumindest auch einen abstrakten Gefahrenbegriff aufweisen. 12 Vorschriften waren nicht eindeutig zuzuordnen, da die Rechtsprechung zu der spezifischen Gefahrenproblematik bislang noch nicht Stellung genommen hat.

Die Unterschiede in der Rechtsprechung werden an einem Beispiel deutlich, das aus dem Regelungsbereich des $§ 70$ StVollzG (Gegenstände zur Freizeitbeschäftigung) stammt. In einer Entscheidung des OLG Zweibrücken aus dem Jahr 1981 (OLG Zweibrücken ZfStrVo 1981, S. 124f.) heißt es: „Gegenstände, die wegen ihres Aussehens, ihrer Beschaffenheit oder Verwendbarkeit die naheliegende Gefahr des Mißbrauchs unter den Gesichtspunkten der Sicherheit oder Ordnung in sich bergen, darf der Gefangene nicht besitzen und zwar unabhängig davon, ob Umstände vorliegen, welche die Gefahr eines Mißbrauchs gerade in der Person dieses Gefangenen nahelegen." Dagegen befindet das OLG Celle (ZfStVo 1983 S. 192), dass $\S 70$ Abs. 2 Nr. 2 StVollzG ,eine konkrete Gefahr für die Sicherheit oder Ordnung der Anstalt voraussetzt. Eine lediglich abstrakte Gefährdung genügt nicht.“ Der Grund für diese unterschiedliche Rechtsprechung ohne Vorlage zum BGH liegt zumindest auch in den unterschiedlichen Sachverhalten: Beim OLG Zweibrücken war es ein Kassettenrecorder, beim OLG Celle eine Schreibmaschine. Ein Hauptproblempunkt, gerade in der Begründung durch die Gerichte, ist der potentiell gefährliche Gegenstand in der Hand eines Gefangenen, bei dem keinerlei konkrete Anhaltspunkte für einen Missbrauch vorliegen. Hier ist das Problem des Missbrauchs durch Mitgefangene zu berücksichtigen. Das Bundesverfassungsgericht hat sich mittlerweile mehrfach der Thematik angenommen (z. B. BVerfG ZfStrVo 97, 172ff. und ZfStrVo 94, 376f.). Obwohl die Verfassungsbeschwerden nicht zur Entscheidung angenommen wurden, erfolgten umfangreichere Ausführungen zur Verfassungskonformität eines abstrakten Gefahrenbegriffes in $§ 70$ StVollzG. Eindeutig äußert sich das Bundesverfassungs- 
gericht, wenn es dem vorentscheidenden OLG Rostock entgegenhält, es habe die ,abstraktgenerelle-Gefahr“ für die Schutzgüter des § $70 \mathrm{StVollzG}$ verkannt.

Die Termini „Gefahr“ und „befürchten“ des Strafvollzugsgesetzes liegen im Zentrum des Spannungsfeldes von Sicherheits- und Behandlungsinteresse einerseits und Gefangenenrechten andererseits. Durch diese Begriffe soll gleichsam ein Austarieren zwischen den beiden Polen erreicht werden. Insgesamt ist die Dominanz eines konkreten Gefahrenbegriffes festzustellen. Was den Begriff des „Befürchtens“ angeht, so wird dieser überwiegend mit der Gefahr gleichgesetzt. Dies trifft für Schrifttum, Rechtsprechung und Vollzugsverwaltung zu. Verwendet wird dieser Begriff vor allem für die schwierigen, von zahlreichen Faktoren beeinflussten Prognosen nach $\S \S 10,11$ und 13 StVollzG. Zudem wird der Begriff "befürchten” immer mit konkretem Gefahrenbezug gebraucht.

Ein dringender Handlungsbedarf für den Gesetzgeber mit Blick auf die Beseitigung möglicher Ungleichbehandlungen, etwa durch eine Erweiterung der Vorlagepflicht der Oberlandesgerichte, ist jedenfalls solange nicht gegeben, wie das Bundesverfassungsgericht im Rahmen der Kontrolle und Wahrung der Grundrechte um eine Abmilderung bemüht ist. Zu wünschen wäre gleichwohl, dass die Oberlandesgerichte mehr Entscheidungen dem Bundesgerichtshof vorlegen und damit ein Stück mehr Rechtsvereinheitlichung und Gleichbehandlung in den verschiedenen Ländern schaffen. Gleiches gilt für die Abstimmung in den Konferenzen der Anstaltsleiter sowie zwischen den einzelnen Justizministerien. Dadurch kann die aufgezeigte Problematik der Ungleichbehandlung sicherlich nicht völlig vermieden, aber zumindest auf ein vertretbares Maß reduziert werden.

Oliver Brinkmann stellte sein Dissertationsvorhaben vor, in dem kriminologische Aspekte im Werk von Alfred Adler aufgezeigt werden sollen. Zwar ist die Individualpsychologie, die der Wiener Mediziner und Psychotherapeut Adler am Anfang des letzten Jahrhunderts entwickelte, in Deutschland und in Euro- 
pa fast vollständig in Vergessenheit geraten - lediglich in der Erziehungswissenschaft spielt die Theorie noch eine Rolle -, in den USA ist der Ansatz aber verhältnismäßig populär. Bei der Entwicklung seiner Theorie bediente sich Adler einer klaren und wenig wissenschaftlichen Sprache, die ihn einerseits für den Laien leicht verständlich machte, ihm andererseits aber den Ruf eines Populärwissenschaftlers einbrachte. Insbesondere Sigmund Freud lehnte den Ansatz ab. Tatsächlich widersprechen sich die Vorstellungen Adlers und die Freuds deutlich. Während Freud daran festhielt, dass biologische und familiäre Faktoren auf dem Gebiet der Psychologie die eigentlichen Grundbedingungen seien, sind für Adler alle Faktoren, denen der Mensch im Laufe seines Lebens und vor allem seiner Kindheit ausgesetzt ist, keine Ursachen für sein Verhalten, sondern lediglich Bedingungen, auf die der Mensch mit seinem Verhalten eine individuelle und schöpferische Antwort gibt. Diese schöpferische Reaktion auf die Lebensbedingungen heißt bei Adler „Lebensstil““. Er ist ausgerichtet auf die Überwindung eines Minderwertigkeitsgefühls, das jedes Kind auf Grund seiner Hilflosigkeit unausweichlich entwickelt. Das Minderwertigkeitsgefühl ist jedoch in der Konzeption der Individualpsychologie kein Schaden der Persönlichkeit, sondern vielmehr Triebfeder und Ansporn für Entwicklung und Verbesserung. Die durch das Minderwertigkeitsgefühl entstehende Unsicherheit wirkt als fortwährender Reiz, um die Anpassung an die Anforderungen des Lebens bewerkstelligen zu können, und ruft ein Streben des Individuums hervor, das Adler „Geltungsstreben“ nennt und das auf die Kompensation des Minderwertigkeitsgefühls im Wege einer Bestätigung des eigenen Selbstwerts gerichtet ist.

Da ein Kind das Rezept für eine gelungene, dauerhafte Kompensation des Minderwertigkeitsgefühls kaum kennen kann, ist es auf seine kreativen Kräfte angewiesen. Diese können sich an Leitlinien orientieren, die durch die Gemeinschaft dem Individuum vorgegeben werden. Die Beeinflussung durch die Gemeinschaftsvorstellungen kann jedoch nur funktionieren, wenn das 
Kind bereits ein Gemeinschaftsgefühl besitzt. Dieses kann nach Adler durch eine entsprechende Erziehung entwickelt werden. Das Gemeinschaftsgefühl ist ein Korrektiv des Geltungsstrebens, indem es die diesem Streben eigene Dynamik zugunsten der Mitmenschen modifiziert. Mangelndes Gemeinschaftsgefühl führt zu mangelnder Kontrolle über das Geltungsstreben und dieses kann sich dann gegen die Interessen der Mitmenschen richten. Fehlt der Blick auf den Mitmenschen, so erfolgt eine Verschiebung der Lebensziele auf die „unnützliche Seite des Lebens“, der Lebensstil des Individuums gestaltet sich auf der Suche nach einer Kompensation des Minderwertigkeitsgefühls als gegen die Gemeinschaft gerichtet. Minderwertigkeitsgefühl, Gemeinschaftsgefühl und Lebensstil sind somit die zentralen Begriffe der Adlerschen Individualpsychologie.

Kriminalität ist aus Sicht der Individualpsychologie ein sozialer Fehlschlag, weil das zugrunde liegende Verhalten lediglich der persönlichen Genugtuung und Bereicherung förderlich ist und dadurch die Gemeinschaft in ihrer Entwicklung stört. Da die Individualpsychologie davon ausgeht, dass jedem Verhalten des Individuums ein entsprechender Lebensstil zugrunde liegt, sieht sie auch kriminelles Verhalten auf einem entsprechenden Lebensstil gegründet. Dies eröffnet den Blick auf Berührungspunkte mit Lebensstil-Ansätzen in der Kriminologie. Sowohl in der Kriminologie als auch in der Individualpsychologie werden Lebensstile als holistische Konzepte verstanden, die das Individuum in seiner Gesamtheit berücksichtigen.

Die starke Bedeutung des Gemeinschaftsgefühls als Korrektiv des Geltungsstrebens erinnert an die Social Control Theorie von Reckless (1961). Dieser verwendet eine der Individualpsychologie ähnliche Begrifflichkeit. Der „innere Halt“ - das Selbstkonzept, die Selbsteinschätzung - und der „äußere Halt“ - das Identitäts- oder Zugehörigkeitsgefühl - sind nach diesem Ansatz zentrale kausale Faktoren für kriminelles Handeln. Auch die Bindungstheorie von Hirschi (1969) weist ein der Adlerschen Vorstellung ähnliches Konzept auf: Die Billigung und Anerkennung des zentralen Wertesystems der Gesellschaft, die Einbindung in gesellschaftliche Gruppen sowie das Verpflichtetsein zur Einhaltung allgemein anerkannter Normen sollen als integrative Elemente des Verhaltens das Straffälligwerden erheblich erschweren. 
Ein mangelhaft ausgebildetes Gemeinschaftsgefühl sorgt für eine mangelhafte Verankerung der die Gemeinschaft tragenden Normen bei dem betroffenen Individuum, also für eine individuelle anomische Situation im Durkheimschen Sinn (Durkheim 1973 und 1988). Dieser versteht Anomie als Regellosigkeit in der Gesellschaft. Somit behandeln Durkheim und Adler ähnliche Phänomene, allerdings auf unterschiedlichen Ebenen. Auch zu Mertons Version der Anomietheorie ist eine Parallele erkennbar. Ähnlich wie Merton (1995) beschreibt auch Adler eine Verhaltenstypologie, wobei sich bei ihm die vorgestellten Typen aus dem Zusammenspiel unterschiedlicher Grade von Gemeinschaftsgefühl und Aktivität der persönlichen Lebensführung ergeben. Kriminelles Verhalten, das bei Merton innovative Rollenanpassung als Reaktion auf den Zustand der Anomie ist, ergibt sich bei Adler aus einem hohen Grad an Aktivität bei gleichzeitig gering ausgebildetem Gemeinschaftsgefühl. Mertons Ansatz ist jedoch der individualistischen Sichtweise Adlers entgegengesetzt. Denn hier sind es die gesellschaftliche Bedingungen, die den einzelnen zum Abweichler werden lassen und nicht individuelle Faktoren. Auch zur Neutralisierungthese von Sykes und Matza (1968) weist der Adlersche Ansatz Berührungspunkte auf.

Insgesamt gesehen steht der Ansatz von Adler in frappierend engem $\mathrm{Zu}$ sammenhang mit einigen bekannten Kriminalitätstheorien. Dies unterstreicht die wissenschaftliche Leistung und Bedeutung Adlers, die im Gegensatz zu seiner verloren gegangenen Bekanntheit steht. Der kriminologische Nutzen der Individualpsychologie könnte in der Ergänzung gängiger Kriminalitätstheorien liegen. Allerdings sind auch Defizite des Ansatzes von Adler erkennbar. Dazu gehört die fehlende umfassende empirische Überprüfung des individualpsychologischen Konzepts, das vor allem auf der eigenen Erfahrung Adlers als Therapeut basiert. 


\section{Literatur}

Albrecht, Hans-Jörg: Die generalpräventive Effizienz von strafrechtlichen Sanktionen. In: Forschungsgruppe Kriminologie (Hrsg.): ,Empirische Kriminologie. Ein Jahrzehnt kriminologischer Forschung am Max-Planck-Institut Freiburg im Breisgau“; Freiburg 1980, S. 305-327.

Bishop, Donna M.: Deterrence and Social Control: A Longitudinal Study of the Effects of Sanctioning and Social Bonding on the Prevention of Delinquency; Albany 1982.

Bishop, Donna M.: Deterrence: A Panel Analysis. In: Justice Quarterly 1984, S. 311-328.

Bock, Michael: Zur dogmatischen Bedeutung unterschiedlicher Arten empirischen Wissens bei prognostischen Entscheidungen im Strafrecht. In Frisch, Wolfgang; Vogt, Thomas (Hrsg.): „Prognoseentscheidungen in der strafrechtlichen Praxis“; Baden-Baden 1994, S. 143-161.

Dölling Dieter: Die Zweiteilung der Hauptverhandlung - Eine Erprobung vor Einzelrichtern und Schöffengerichten; Göttingen 1978.

Dölling, Dieter u.a.: Die Dauer von Strafverfahren vor den Landgerichten. Eine empirische Analyse zur Rechtswirklichkeit von Strafverfahren in der Bundesrepublik Deutschland; Köln 2000.

Durkheim, Emile: Der Selbstmord; Neuwied, Berlin 1973.

Durkheim, Emile: Über soziale Arbeitsteilung. Studie über die Organisation höherer Gesellschaften; Frankfurt/M. 1988, 2. Aufl.

Eisele, Hermann: Die general- und spezialpräventive Wirkung strafrechtlicher Sanktionen. Methoden, Ergebnisse, Metaanalyse; Dissertation, Universität Heidelberg, Heidelberg 1999.

Erickson, Maynard L.; Gibbs, Jack P.; Jensen, Gary F.: The Deterrence Doctrine and the Perceived Certainty of Legal Punishments. In: American Sociological Review 1977, S. 305317. 
Frisch Wolfgang: Prognoseentscheidungen im Strafrecht - zur normativen Relevanz empirischen Wissens und zur Entscheidung bei Nichtwissen; Heidelberg, Hamburg 1983.

Göppinger, Hans: Angewandte Kriminologie. Ein Leitfaden für die Praxis; Berlin, Heidelberg 1985.

Hirschi, Travis: Causes of Delinquency; Berkeley 1969.

Lamnek, Siegfried: Neue Theorien abweichenden Verhaltens; Frankfurt/M. 1994.

Merton, Robert K.: Sozialstruktur und Anomie. In: Meja, V./ Stehr, N. (Hrsg.): „Robert K. Merton. Soziologische Theorie und soziale Struktur“, Berlin, New York 1995, S. 127-154.

Reckless, Walter C.: Halttheorie. In: MschrKrim 1961, S. 1-14.

Rehn, Gerhard: Behandlung im Strafvollzug. Ergebnisse einer vergleichenden Untersuchung der Rückfallquote bei entlassenen Strafgefangenen; Weinheim, Basel 1979.

Streng, Franz: Strafrechtliche Folgenorientierung und Kriminalprognose. In Dölling, Dieter (Hrsg.): „Die Täter - Individualprognose. Beiträge zu Stand, Problemen und Perspektiven der kriminologischen Prognoseforschung“; Heidelberg 1995, S. 97 - 127.

Sykes Gresham M.; Matza, David: Techniken der Neutralisierung: eine Theorie der Delinquenz, in: Sack, Fritz; König, Rene (Hrsg.): „Kriminalsoziologie“, Frankfurt/Main 1968, S. 360-371.

Anschrift:

Dipl-Päd. Horst Beisel, Prof. Dr. Dieter Dölling und Priv.-Doz. Dr. Dieter Hermann, Institut für Kriminologie, Friedrich-Ebert-Anlage 6-10, Universität Heidelberg, 69117 Heidelberg, Tel. 06221-547489/-90 (Sekretariat), Fax: 06221-54 7495,

E-Mail: ifk@krimi.uni-heidelberg.de 